\title{
Postural Modes and Control for Dexterous Mobile Manipulation: the UMass uBot Concept
}

\author{
Dirk Ruiken and Michael W. Lanighan and Roderic A. Grupen \\ Laboratory for Perceptual Robotics \\ School of Computer Science \\ University of Massachusetts Amherst \\ Amherst, MA 01003, USA \\ \{ruiken, lanighan, grupen\}ecs.umass.edu
}

\begin{abstract}
We present the UMass uBot concept for dexterous mobile manipulation. The uBot concept is built around Bernstein's definition of dexterity - "the ability to solve a motor problem correctly, quickly, rationally, and resourcefully" [1]. We contend that dexterity in robotic platforms cannot arise from control alone and can only be achieved when the entire design of the robot affords resourceful behavior. uBot-6 is the latest robot in the uBot series whose design affords several postural configurations and mobility modes. We discuss these dexterous mobility options in detail and demonstrate the strength of dexterous mobility.
\end{abstract}

\section{INTRODUCTION}

Mobile manipulation in unstructured environments is the focus of a great deal of current and ongoing research [2], [3], [4], [5], [6]. Among the most important technical challenges concerns the generation of contingencies for situations that are not necessarily anticipated beforehand and for dealing with generic constraints imposed by different tasks. In order to succeed in such situations a robot needs to have a wide variety of skills at its disposal. Traditionally, these skills are either acquired autonomously in a constrained setting or preprogrammed. Mobility is often just a means of extending reach. However, slight variations of the task or the environment may render a single solution ineffective. Therefore, a robot must be able to repair parts of its integrated skills that are sensitive to run-time variation efficiently using functionally equivalent (sub)skills that are appropriate. We present a mobile manipulator concept that incorporates dexterity in both mobility and manipulation in order to become robust to variations in the environment or task.

In the seminal work "On Dexterity and Its Development" by Nikolai Bernstein, dexterity is described as "the ability to solve a motor problem correctly, quickly, rationally, and resourcefully" [1]. This definition applies equally to mobility as it does to manipulation and it makes it clear that dexterity must be the product of the entire design-it does not simply emerge from control alone. The focus of this paper is on the "resourcefulness" specification. In this case, "dexterity" implies that when a prototype solution cannot be applied due to run-time constraints, a robot can modify its strategy quickly to deal with the unexpected circumstances. Our approach is to design mechanical systems with different types of solutions with varying performance characteristics that can be exploited when required.

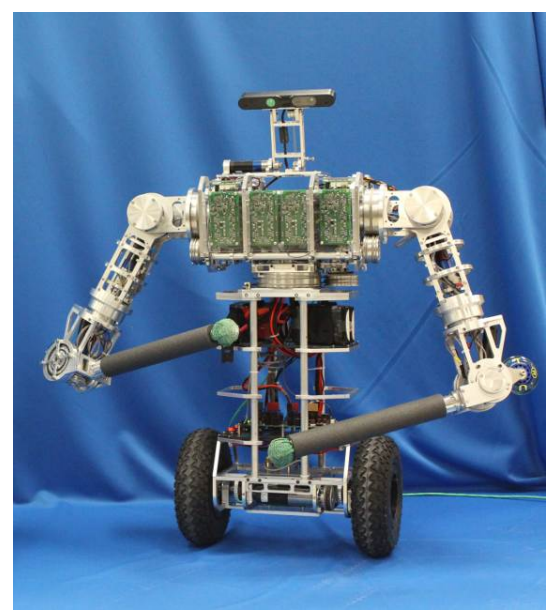

Fig. 1. uBot-6: a 12 degree of freedom whole body mobile manipulator

We present a comprehensive design for dexterous mobility and postural control using the uBot-6. Section II describes platforms that achieve some level of dexterous mobility. In Section III we provide details about the uBot-6 mobile manipulator. Postural options for uBot-6 are described in Section IV. uBot-6's mobilty options arising from available postural modes are described in Section V. Section VI presents some demonstrations to showcase new forms of mobility in uBot-6 and characterize their performance. Finally, Section VII describes future research goals.

\section{RELATED WORK}

There are very few robotic platforms that use dynamic reassignment of effectors from manipulation to mobility tasks and vice versa. The NASA Athlete [7] can reuse its legs to perform manipulation tasks. RobotCub's iCub can walk using just the legs [8] and crawl using both arms and legs [9]. Tbot [10] is a dynamically balancing platform which can transform into a statically stable four wheel drive vehicle. While it mounts wheels to 'arms' for this purpose, these effectors are not suited or intended for manipulation. The Vecna Bear [11] has multiple mobility options at its disposal. It can reconfigure its tracks in many ways allowing it to overcome various terrain types and even stand up tall balancing dynamically. Using a completely 
different approach, modular robots such as SuperBot [12] can reconfigure to accommodate a particular mobility task. In all these cases the traditional barrier between mobility and manipulation has been diminished leading to increased performance.

The previous versions of uBot also possessed limited capabilities of dynamically reassigning effectors. Thibodeau et al. [13] have shown superior manipulation performance by using wheel motors and postural control for manipulation tasks. Kuindersma et al. [14] demonstrated improved mobility on the uBot by using the arms for 'knuckle-walking'.

\section{THE UBOT-6 MOBILE MANIPULATOR}

The uBot-6 is a toddler-sized mobile manipulator that balances dynamically on two wheels (Fig. 1). It is being developed at the Laboratory for Perceptual Robotics at the University of Massachusetts Amherst as part of a series of mobile manipulators. Experience from the design and operation of uBot-4 [15], [13] and uBot-5 [14] has been incorporated into the design of uBot-6. Compared to uBot-5, there has been at least a threefold increase in strength of all joints.

The robot has 12 degrees of freedom (DOF): two wheels, a rotatable trunk with two 4-DOF arms, and a 1-DOF head. Added in uBot-6, the elbows are equipped with small, unactuated wheels that enable additional forms of mobility. The basic physical dimensions of the uBot- 6 robot compared to its predecessor uBot-5 are summarized in Table I.

TABLE I

BASIC SPECIFICATIONS OF THE UBOT-5 AND UBOT-6 ROBOTS.

\begin{tabular}{|l|c|c|}
\hline & uBot-5 & uBot-6 \\
\hline \hline Body height (to top of head) & $77 \mathrm{~cm}$ & $88 \mathrm{~cm}$ \\
\hline Body width & $59 \mathrm{~cm}$ & $60 \mathrm{~cm}$ \\
\hline Body depth & $20 \mathrm{~cm}$ & $23 \mathrm{~cm}$ \\
\hline Body mass & $18.9 \mathrm{~cm}$ & $24.8 \mathrm{~kg}$ \\
\hline Arm length (shoulder to hand) & $57 \mathrm{~cm}$ & $73 \mathrm{~cm}$ \\
\hline Base wheel diameter & $20.5 \mathrm{~cm}$ & $23 \mathrm{~cm}$ \\
\hline Elbow wheel diameter & - & $7 \mathrm{~cm}$ \\
\hline
\end{tabular}

A new 1-DOF head (Fig. 2) is designed to complement the postural versatility of the robot: two coupled joints driven by one motor result in a combined rotational and translational movement that permits full view of the bimanual workspace in all postural modes. An RGB-D camera (Asus Xtion Pro Live) provides color and depth information from the manual workspace.

High performance low-level motor control at $2 \mathrm{KHz}$ is provided by a custom FPGA board. An on-board quadcore computer runs Linux and ROS [16] Fuerte to implement mobility and manipulation controls as well as vision processing and behavioral programs. Wireless connectivity allows additional computation to be performed on external computers as well as communication between several robots.

\section{WHOLE BODY POSTURAL CONTROL}

The uBot-6 robot is designed for whole body dexterous mobility and manipulation. It can assume several postural

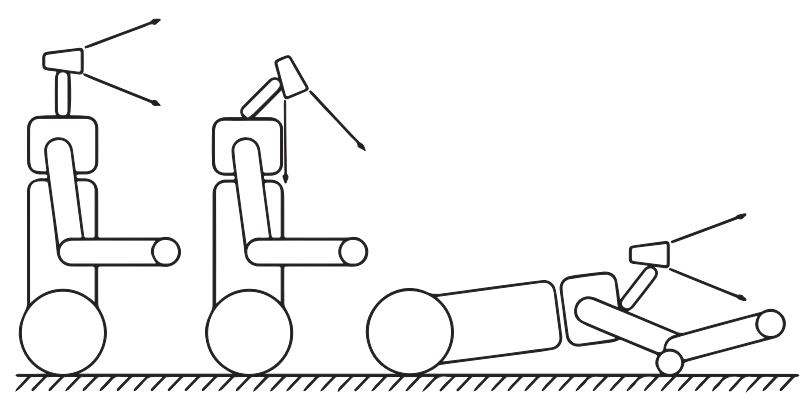

Fig. 2. uBot-6 head: one motor drives two coupled degrees of freedom resulting in a combined rotation and forward-backward translation. This mechanism permit full view of the bimanual workspace in all body postures.

configurations that afford different capacities for mobility and manipulation (see Fig. 4).

\section{A. Prone}

In this configuration the robot lies on the anterior surface of its body. In this postural mode, trunk rotation is disabled and both arms are free with a limited overall reachable workspace, a significant portion of which is in the ground plane. The robot footprint is relatively large in this configuration and the body height is minimized.

\section{B. 4-Point Stance}

In this stance, the base wheels as well as both arms are in contact with the ground. The arm ground contact is established with hands and/or elbow wheels. In case of ground contact with the elbows, the hands can still be moved in the nullspace and used for manipulation actions. This posture allows the body height to vary between $24 \mathrm{~cm}$ and $88 \mathrm{~cm}$.

\section{3-Point Stance}

This stance is a quasi-statically stable tripod with both base wheels and one arm in contact with the ground (Fig. 3). The arm ground contact is established either with a hand or an elbow wheel. There are an infinite number of ways to achieve this configuration by varying the choice of left or right arm, hand or elbow ground contact, body height, and location of the ground contact. The choice of body height and ground contact location are constrained by the stability requirement of keeping the zero moment point (ZMP) inside the triangle formed by the three ground contacts [17], [14].

The second arm is free for manipulation actions and only constrained by the stability requirement. Therefore, only movements that do not shift the ZMP out of the support triangle are allowed. As in the case of the 4-point stance, the body height can be varied between $24 \mathrm{~cm}$ and $88 \mathrm{~cm}$.

\section{2-Point Stance}

The robot stands upright by balancing on its two base wheels (see Fig. 1). A Linear Quadratic Regulator (LQR) runs on the onboard FPGA and controls the balancing at $1 \mathrm{KHz}$. In this postural configuration the robot has a minimal ground footprint. The dynamic stability of this posture results 


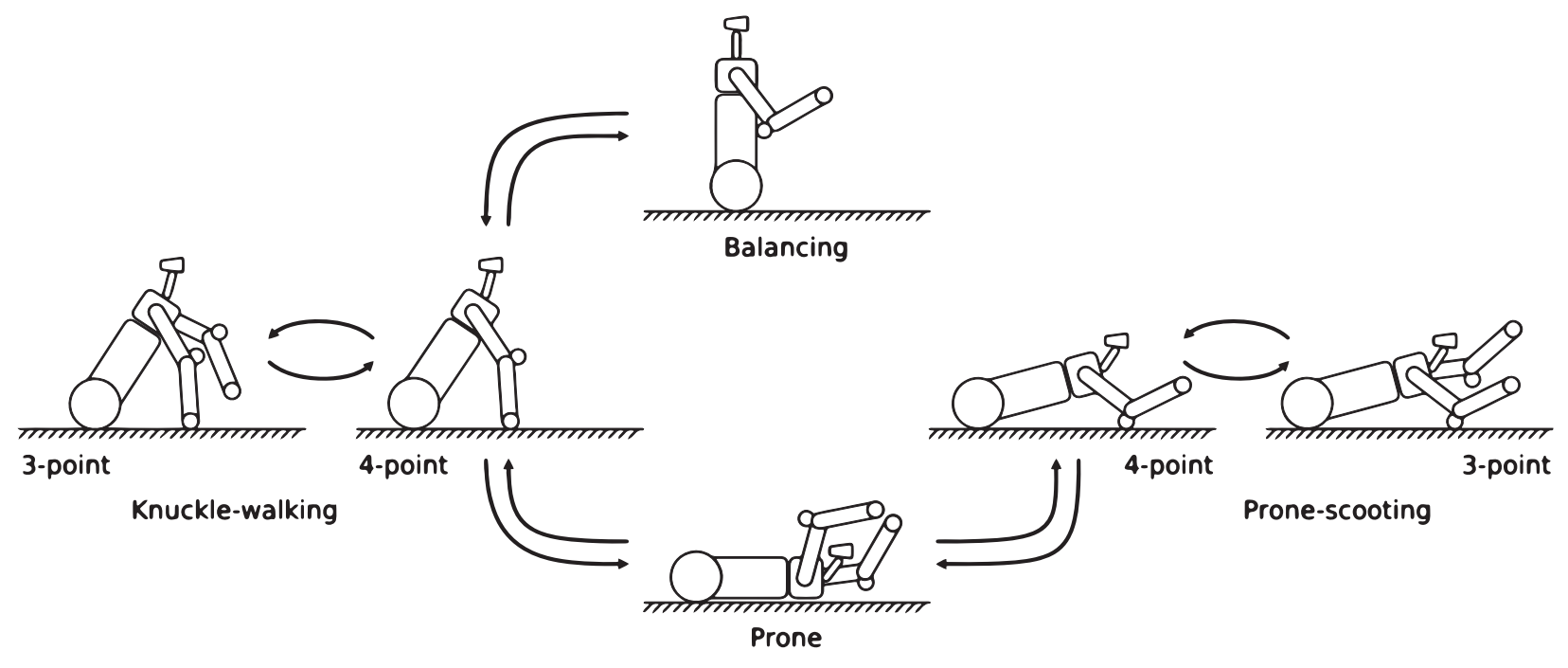

Fig. 4. Postural transition graph for the uBot-6. Postural options include: 2-point contact during dynamic balancing, 3 and 4-point contact during knuckle-walking and prone scooting, and prone resting.

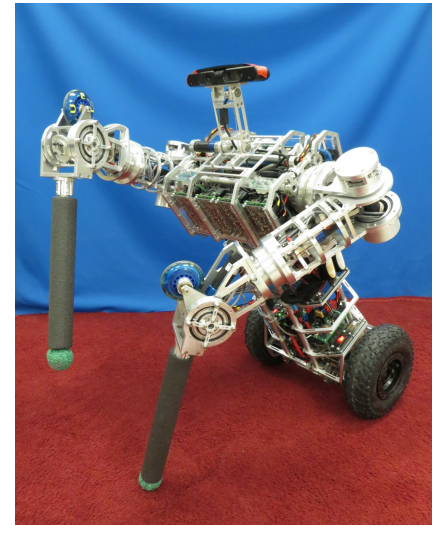

Fig. 3. A 3-point postural configuration of uBot-6.

in low longitudinal input impedance. This contributes to safety in the presence of humans.

In this posture, the arms of the robot are free to perform manipulation tasks. Trunk rotation and the symmetric workspace of the arms contribute to a large bimanual workspace. This includes a large area on the ground plane while providing accessibility to counter tops and low shelves. uBot-6 uses a forward compensator to anticipate the effect of its own upper body posture on the center of mass in order to improve the end point precision of the robot.

\section{E. Postural Transitions}

The robot can reliably transition between the different postural configurations by following a path through the postural transition graph as depicted in Figure 4. Table II shows the time and energy cost associated with each available transition. Additional transitions such as the transition from a 4-point-hand stance to a 4-point-elbow stance without passing through prone configuration can easily be imagined.
Implementation details for these controllers can be found in Kuindersma et al. [14].

TABLE II

TIME AND ENERGY REQUIRED TO EXECUTE A TRANSITION. ${ }^{1}$

\begin{tabular}{|c|c|c|}
\hline & $\begin{array}{c}\text { Mean energy } \\
\text { used (J) }\end{array}$ & $\begin{array}{c}\text { Mean execution } \\
\text { time (s) }\end{array}$ \\
\hline 2-point to 4-point (hand) & $188.43 \pm 5.11$ & $5.26 \pm 0.07$ \\
\hline 4-point (hand) to 2-point & $260.07 \pm 7.96$ & $6.03 \pm 0.03$ \\
\hline 4-point (hand) to Prone & $631.57 \pm 84.52$ & $9.59 \pm 0.09$ \\
\hline Prone to 4-point (hand) & $981.42 \pm 71.05$ & $10.04 \pm 0.11$ \\
\hline 4-point (elbow) to Prone & $118.10 \pm 51.35$ & $2.25 \pm 0.97$ \\
\hline Prone to 4-point (elbow) & $165.11 \pm 12.77$ & $4.20 \pm 0.10$ \\
\hline
\end{tabular}

\section{DEXTEROUS MOBILITY}

The uBot has multiple forms of mobility at its disposal. Each such mobility mode offers unique possibilities to move in various environments. In this section, we highlight and characterize three of those mobility modes: balancing mobility, knuckle-walking, and prone-scooting. Time and energybased cost models for these modes based on empirical data are presented. Each mobility mode uses one or more postural configurations for support. Other possible mobility modes such as elbow-walking are left for future work.

\section{A. Balancing Mobility}

Balancing mobility combines dynamic balancing in 2point stance with differential steering. The wheels are controlled by an LQR that maintains the robot's balance while performing translational and rotational movements. The steering geometry allows uBot to rotate in place. This combined with the small 2-point footprint enables navigation in tight spaces. Dynamic balancing is very energy efficient as

\footnotetext{
${ }^{1}$ In the future, different motor drivers with the capacity for regenerative damping will allow the robot to recover energy in transitions to postural configurations with lower potential energy.
} 
only small compensation movements have to be performed. While standing in place, the wheels require about $2.6 \mathrm{~W}$ to balance. Figure 5 shows the variation in power consumption of the wheel motors with drive speed. The maximum drive speed and turnrate in this mobility mode is limited to $1.2 \frac{\mathrm{m}}{\mathrm{s}}$ and $4.1 \frac{\mathrm{rad}}{\mathrm{s}}$ respectively. Additional torque is reserved for compensatory balancing movements.

Base Power Consumption for Balancing Mobility and Prone Scooting

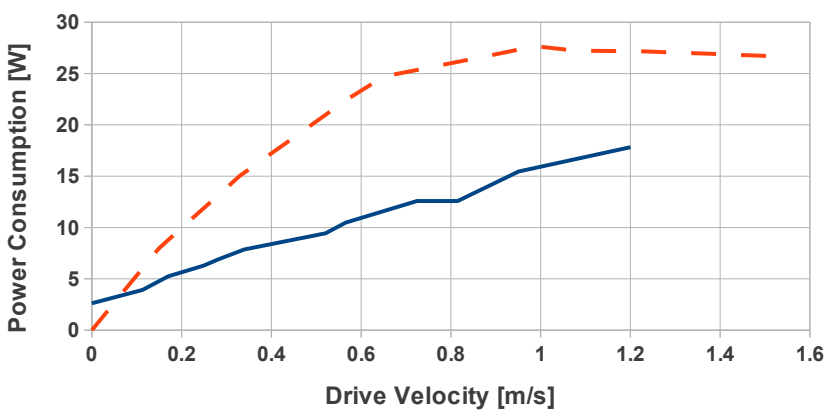

Fig. 5. Power consumption [W] of the wheel motors over drive speed $\left[\frac{\mathrm{m}}{\mathrm{s}}\right]$ in balancing mobility (solid line) and prone scooting (dashed line). Pure balancing while standing consumes $2.6 \mathrm{~W}$. It should be noted that while prone scooting the power consumption is dominated by the arms with additional $20-80 \mathrm{~W}$ depending on the body height.

\section{B. Prone Scooting}

In this mobility mode, the robot is configured in a 4point-elbow postural stance in which the base wheels and the two small wheels attached to the elbows are in contact with the ground (Fig. 6). In this mode, the robot drives like a car. However, the steering is more complicated since the front wheels are attached to the elbows and all four arm joints impact the driving behavior. Three shoulder joints with angles $\theta_{1}, \theta_{2}$, and $\theta_{3}$ determine both the location and orientation of the elbow wheel for each arm. Figure 7 shows the robot while in prone scooting configuration. Drive inputs include the desired body height, drive velocity, and steering angle.

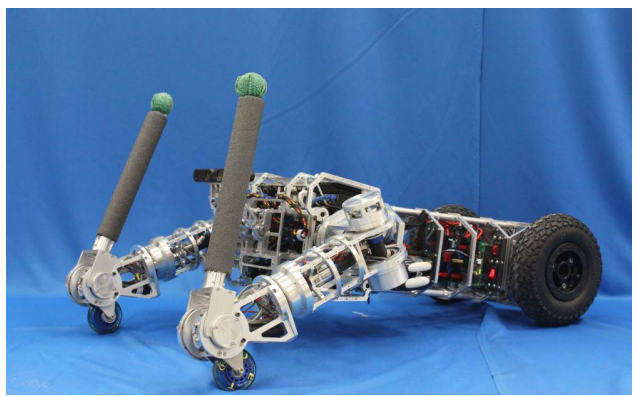

Fig. 6. One of many configurations for prone scooting. Depending on the available height and required turn radius, the robot can adjust its prone scooting configuration. The forearms remain free for manipulation movements in the nullspace of the Ackermann steering.

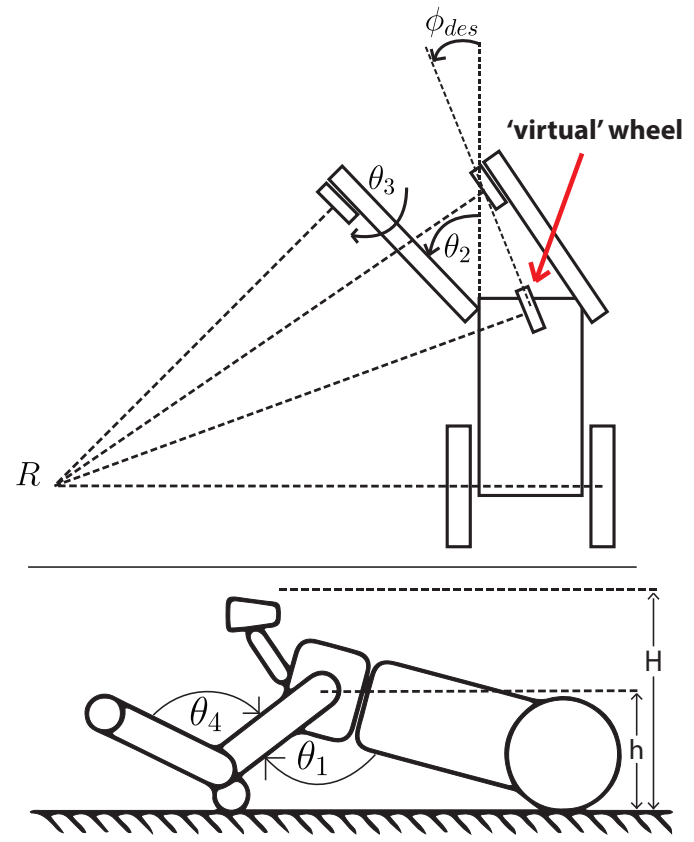

Fig. 7. Prone scooting mobility - Top: the desired steering angle $\phi_{\text {des }}$ is used to orient a 'virtual' wheel located midway between the shoulders. The intersection of the axes of base wheels and the 'virtual' wheel determines the center of rotation $R$. Control of shoulder joint angles $\theta_{2}$ and $\theta_{3}$ allows this turn radius to be matched with both front wheels to avoid slip. Bottom: shoulder angle $\theta_{1}$ and elbow angle $\theta_{4}$ determine shoulder height $h$ and body height $H$.

1) Body Height: In this mobility mode the body height $H$ of the robot is determined by the height of the shoulders $h$ as well as the pose of the forearms. The height of the shoulder is primarily determined by the first shoulder joint angle $\theta_{1}$. We neglect the minimal influence of $\theta_{2}$ and $\theta_{3}$ in order to simplify control. Given a desired body height the necessary shoulder height is calculated by subtracting a safety distance of $12 \mathrm{~cm}: h=H-0.12 m$. The required shoulder joint angle $\theta_{1}$ is given by

$$
\theta_{1}=\operatorname{acos}\left(\frac{h-r_{1}}{b}\right)+\operatorname{acos}\left(\frac{h-r_{2}}{a}\right)
$$

where $a$ is the upper arm length, $b$ is the distance from base wheel axis to shoulder, and $r_{1}$ and $r_{2}$ are the wheel radii of base and elbow wheels respectively. The elbow joint angle $\theta_{4}$ only influences the body height if the forearm protudes higher than the body. In this mode, the body height can be varied between $24 \mathrm{~cm}$ (in which the robot is almost completely prone) and $46 \mathrm{~cm}$.

2) Steering Angle: Scooting requires Ackermann steering where all wheel axes must intersect at a common center of rotation (Fig. 8). However, unlike cars, the arm joints influence the location and orientation of the wheel axes of the front wheels. The wheel axes are not guaranteed to be parallel to the ground plane any more. Therefore, instead of the wheel axes we use their projection onto the ground plane throughout the rest of this section.

In order to convert the steering angle input into a turn 
radius, we define a 'virtual' wheel midway between both shoulders following Cholet et al. [18]. The steering angle input specifies its orientation $\phi_{\text {des }}$ (see Fig. 7 top). The intersection between wheel axes of 'virtual' wheel and base wheels defines the desired center of rotation $R$.

To ensure that the wheels do not slip, the free shoulder angles $\theta_{2}$ and $\theta_{3}$ are controlled such that the wheel axes of the front wheels intersect the desired center of rotation. Both shoulder joints have varying and coupled influence on the location and orientation of the front wheels which depends on both the body height and the current arm configuration. A Jacobian based controller minimizes the orientation error of the front wheels.

Given the steering angles $\phi_{i}$ where $i=1$ denotes the left arm and $i=2$ denotes the right arm, the steering error $\epsilon_{i}=\phi_{\text {des }}-\phi_{i}$ is computed. The orientation constraint of the front wheels is expressed as a quadratic potential function $\epsilon_{i}^{2}$.

The error Jacobian $J_{i}$ that describes the sensitivity of the orientation error with respect to the control variables $\theta_{2}$ and $\theta_{3}$ is given by

$$
J_{i}=\left[\begin{array}{ll}
\frac{\partial \epsilon_{i}^{2}}{\partial \theta_{2}} & \frac{\partial \epsilon_{i}^{2}}{\partial \theta_{3}}
\end{array}\right] .
$$

These Jacobians are calculated numerically and allow us to compute updates for reference inputs of the shoulder joints with

$$
\left[\begin{array}{c}
\Delta \theta_{2} \\
\Delta \theta_{3}
\end{array}\right]=-J_{i}^{\#} \epsilon_{i}^{2}
$$

where $J_{i}^{\#}$ is the pseudoinverse of $J_{i}$ [19]. The resulting closed-loop controller synchronizes both arms such that the desired turn radius is achieved while ensuring that the wheels do not slip.

The minimal turn radius is dependent on the chosen body height. When the robot is at its lowest body height a turn radius of $1.2 \mathrm{~m}$ can be achieved. In this configuration, the steering depends mostly on the second shoulder joint (which is limited to avoid collision with the upper trunk). At its highest body height, a turn radius of $0.68 \mathrm{~m}$ can be achieved. The third shoulder joint is at its joint limit preventing tighter turning. Figure 8 shows the intersection of the wheel axes and minimal turning radius according to Ackermann steering for a low (left) and high (right) body height. The minimal turn radii achievable for varying body heights is shown in Figure 9.

3) Drive Velocity: The desired drive velocity is used to calculate reference velocities for each base wheel. Depending on the desired turn radius of the robot, the base wheels travel on different radii and thus are commanded different velocities in order to prevent slipping. The maximum drive speed is $1.8 \frac{\mathrm{m}}{\mathrm{s}}$.

The energy cost of this mobility mode depends on two factors: the power consumption of the arms to keep the body up and the power consumption of the wheels to drive the robot. The power consumption of the wheel motors is similar to balancing mobility and ranges from $0 \mathrm{~W}$ to $24 \mathrm{~W}$ depending on the velocity. The power consumption of the arms to hold up the body ranges from $32 \mathrm{~W}$ (almost prone) to $16 \mathrm{~W}$ (tallest).

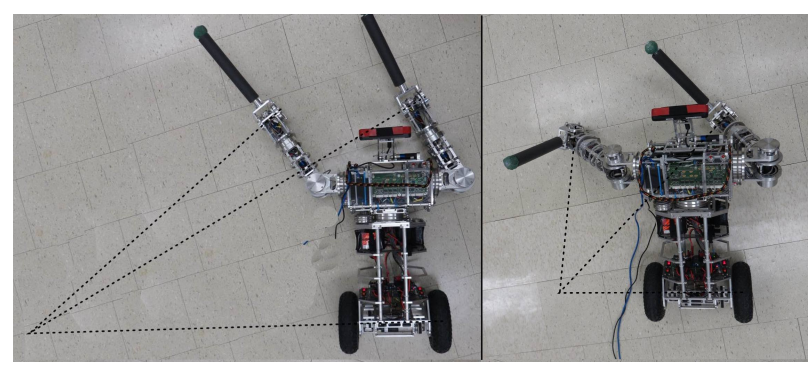

Fig. 8. Maximal turning for two different robot body heights. The dotted lines depict the wheel axes intersecting in the center of rotation. When the body is almost flat on the ground (left) the turn radius is strongly restricted by the joint limits of the arms. With raised body two joints in each arm are used for steering allowing a signicantly smaller turn radius (right).

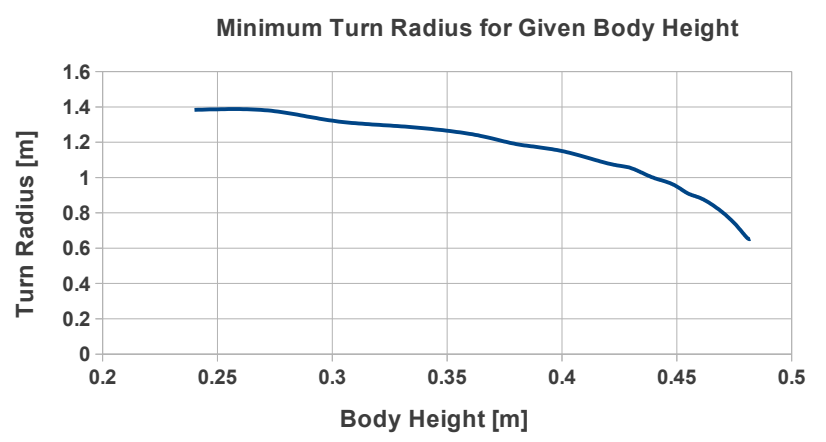

Fig. 9. Dependency of available minimal turn radius from the body height. With increasing body height smaller turn radii can be achieved as different joint combinations are used for steering.

\section{Knuckle-walking}

Knuckle-walking is an upper-limb walking gait that uses the hands as ground contacts. It is based on sequencing of 3-point and 4-point postural configurations. Kuindersma et al. [14] presented a preliminary implementation of this controller for uBot-5. In this work a walking gait was learned efficiently in simulation from primitive controllers.

The knuckle-walking gait provides uBot the ability to traverse otherwise hazardous terrain. This ability comes at the cost of higher energy consumption and slower movement speed compared to locomotion while balancing. The full demonstration fo knuckle-walking on the uBot-6 remains future work.

\section{DEMONSTRATION}

In the following demonstration we show how dexterous mobility can overcome environmental variations that are impossible without multiple postural modes. The demonstration is carried out via teleoperation. The uBot approaches in a 2point balancing stance and transitions to prone to navigate beneath the coffee table. Once it has passed underneath, the robot transitions once again to a vertical posture (see Fig. 10). 


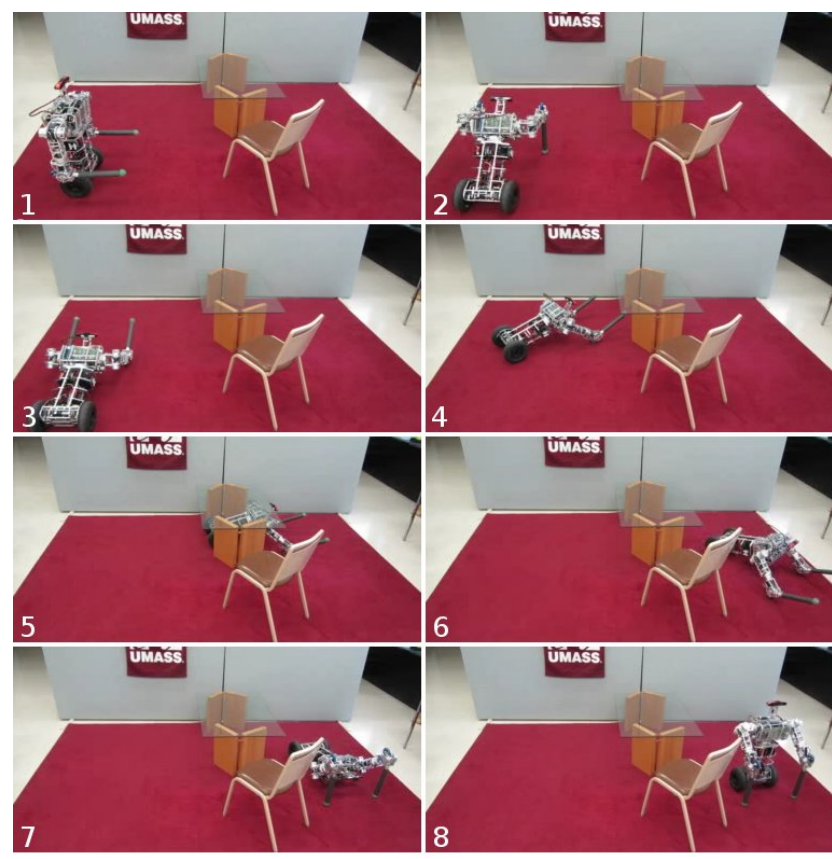

Fig. 10. Demonstration of prone scooting. uBot-6 is able to enter areas of the environment unreachable with balancing mobility. Here a chair and table block the path. After a transition to prone scooting the robot can pass under the table and stand up on the other side.

\section{CONCLUSION}

Robots need to be resourceful to act successfully in unstructured environments. The resourcefulness arises not just by using control, but also by designing hardware that can be reconfigured based on task and environment. We presented the uBot- 6 , a robot suited for mobile manipulation and designed specifically for whole body mobility. We described and characterized several of its mobility modes. We used empirical data to create models for time and energy costs associated with the mobility modes and mode transitions. A teleoperated demonstration showing the advantages of having multiple mobility modes was also presented. These models will enable planning and reasoning when selecting mobility modes for navigation tasks.

Future work includes further devloping the empirical models of costs for autonomous planning and reasoning under uncertainty.

\section{ACKNOWLEDGMENT}

This material is based upon work supported under Grants NASA-GCT-NNH11ZUA001K and ONR-MURIN000140710749.

\section{REFERENCES}

[1] N. A. Bernstein, "On dexterity and its development," in Dexterity and its development, M. L. Latash and M. Turvey, Eds. Mahwah, NJ: Lawrence Erlbaum Associates, 1996, pp. 1-237.

[2] A. Pratkanis, A. E. Leeper, and K. Salisbury, "Replacing the office intern: An autonomous coffee run with a mobile manipulator," in Robotics and Automation, 2013. Proceedings. 2013 IEEE International Conference on, 2013.

[3] E. Marder-Eppstein, E. Berger, T. Foote, B. Gerkey, and K. Konolige, "The office marathon: Robust navigation in an indoor office environment," in Robotics and Automation (ICRA), 2010 IEEE International Conference on. IEEE, 2010, pp. 300-307.

[4] A. Hornung, M. Phillips, E. Jones, M. Bennewitz, M. Likhachev, and S. Chitta, "Navigation in three-dimensional cluttered environments for mobile manipulation," in Robotics and Automation (ICRA), 2012 IEEE International Conference on, 2012, pp. 423-429.

[5] M. Stilman and J. Kuffner, "Navigation among movable obstacles: Real-time reasoning in complex environments," International Journal on Humanoid Robotics, vol. 2, no. 4, pp. 479-504, December 2005.

[6] P. Deegan, R. Grupen, A. Hanson, E. Horrell, S. Ou, E. Riseman, S. Sen, B. Thibodeau, A. Williams, and D. Xie, "Mobile manipulators for assisted living in residential settings," Autonomous Robots, vol. 24, no. 2, pp. 179-192, 2008.

[7] B. H. Wilcox, T. Litwin, J. Biesiadecki, J. Matthews, M. Heverly, J. Morrison, J. Townsend, N. Ahmad, A. Sirota, and B. Cooper, "Athlete: A cargo handling and manipulation robot for the moon," Journal of Field Robotics, vol. 24, no. 5, pp. 421-434, 2007. [Online]. Available: http://dx.doi.org/10.1002/rob.20193

[8] Z. Lu, S. Lallee, V. Tikhanoff, and P. Dominey, "Bent leg walking gait design for humanoid robotic child-icub based on key state switching control," in Robotics and Applications (ISRA), 2012 IEEE Symposium on, June, pp. 992-998.

[9] S. Degallier, L. Righetti, L. Natale, F. Nori, G. Metta, and A. Ijspeert, "A modular bio-inspired architecture for movement generation for the infant-like robot icub," in Biomedical Robotics and Biomechatronics, 2008. BioRob 2008. 2nd IEEE RAS EMBS International Conference on, Oct., pp. 795-800.

[10] T. L. Hutcheson and J. E. Pratt, "Reconfigurable balancing robot and method for dynamically transitioning between statically stable mode and dynamically balanced mode," Patent Application, 05 2008, uS 2008/0105481 A1.

[11] V. Robotics, "the bear battlefield extraction-assist robot," 2010.

[12] W.-M. Shen, M. Krivokon, H. Chiu, J. Everist, M. Rubenstein, and J. Venkatesh, "Multimode locomotion via superbot reconfigurable robots," Autonomous Robots, vol. 20, no. 2, pp. 165-177, 2006.

[13] B. J. Thibodeau, P. Deegan, and R. Grupen, "Static analysis of contact forces with a mobile manipulator," in Robotics and Automation, 2006. ICRA 2006. Proceedings 2006 IEEE International Conference on. IEEE, 2006, pp. 4007-4012.

[14] S. Kuindersma, E. Hannigan, D. Ruiken, and R. Grupen, "Dexterous mobility with the ubot-5 mobile manipulator," in Advanced Robotics, 2009. ICAR 2009. International Conference on, June, pp. 1-7.

[15] P. Deegan, B. J. Thibodeau, and R. Grupen, "Designing a selfstabilizing robot for dynamic mobile manipulation," DTIC Document, Tech. Rep., 2006.

[16] M. Quigley, K. Conley, B. Gerkey, J. Faust, T. Foote, J. Leibs, R. Wheeler, and A. Y. Ng, "Ros: an open-source robot operating system," in ICRA workshop on open source software, vol. 3, no. 3.2, 2009.

[17] M. Vukobratović and J. Stepanenko, "On the stability of anthropomorphic systems," Mathematical Biosciences, vol. 15, no. 1, pp. 1-37, 1972.

[18] H. Choset, K. M. Lynch, S. Hutchinson, G. Kantor, W. Burgard, L. E Kavraki, and S. Thrun, Principles of robot motion: theory, algorithms, and implementation. Bradford Books, 2005.

[19] Y. Nakamura, Advanced Robotics: Redundancy and Optimization. Addison-Wesley, 1991. 